
\title{
28 Research Square \\ Opposite Regulation of F508del-CFTR Biogenesis by Four Poly-Lysine Ubiquitin Chains
}

\section{Qingtian Wu}

Jiamusi University

Yonta Tiakouang Henri

Jiamusi University

\section{Ruixue Yao}

Jiamusi University

Xuemei Ma

Jiamusi University

Lei Liu

Jiamusi University

Nana Noutchie Vinye

Jiamusi University

\section{Xiubin Liang}

Nanjing Medical University

Gang Zhao

Jiamusi University

Xia Hou ( $\square$ houxia@jmsu.edu.cn )

Universite de Lorraine https://orcid.org/0000-0002-6733-086X

\section{Research article}

Keywords: F508del-CFTR, proteasomal degradation, Lysine linked polyubiquitin chain

Posted Date: November 2nd, 2020

DOI: https://doi.org/10.21203/rs.3.rs-98616/v1

License: (c) (i) This work is licensed under a Creative Commons Attribution 4.0 International License.

Read Full License 


\section{Abstract}

Background: As a misfolding protein, more than $99 \%$ of F508del-CFTR (F508del) is degraded by the ubiquitin-proteasome system before its maturation, which results in almost no membrane expression of CFTR and thereby no chloride secretion across epithelial cells of cystic fibrosis patients. The conjugation of ubiquitin chains to the protein substrates is necessary for ubiquitin-mediated proteasomal degradation. Ubiquitin contains seven lysine $(K)$ residues (K6, K11, K27, K29, K33, K48, and K63), all of them can be conjugated one to another forming poly-ubiquitin chains on substrates by mixing together or by only one type of lysine that provides sorting signals for different pathways.

Results: Here we report that four lysine linked poly-Ub chains (LLPUCs) were involved in F508del biogenesis: two LLPUCs linked by K11 or K48 of Ub facilitated F508del degradation; whereas the other two linked by K63 and K33 of Ub protected F508del from degradation. LLPUC K11 is more potent for F508del degradation than K48. Moreover, E3 ligase CHIP and RNF5 catalyzed LLPUCs K48 formation and RNF5 also catalyzed LLPUC K11 formation on F508del. Importantly, Those LLPUCs provide F508del with different affinities to the proteasomal shuttle protein (Rad23a) and the proteasomal receptors (Adrm1 and S5a), offering a mechanistic insight of differential regulation F508del different by different LLPUCs.

Conclusions: F508del utilizes four specific lysine-linked poly Ub chains and during its biogenesis for opposite destiny through different identification by proteasomal shuttle protein or receptors. These findings provide new insights to understand the CF pathogenesis and are expected to facilitate the development of therapies for this devastating disease.

\section{Background}

Cystic fibrosis (CF) is one of the most common recessive autosomal disorders induced by disease causing mutations in the cystic fibrosis transmembrane conductance regulator (CFTR) gene ${ }^{1}$. More than $90 \%$ of CF patients carry a three base pair mutation on at least one allele that leads to the deletion of the phenylalanine residue at position 508 of CFTR (F508del-CFTR) ${ }^{2,3}$. As a misfolded protein, almost all F508del-CFTR (F508del) is degraded by the ubiquitin proteasome system (UPS) before its maturation, which results in almost no CFTR membrane expression and thereby no chloride secretion across epithelial cells of CF patients ${ }^{4}$.

Ubiquitin (Ub), a highly conserved 76 amino acid polypeptide, contains seven lysine (K) residues (K6, K11, $\mathrm{K} 27, \mathrm{~K} 29, \mathrm{~K} 33, \mathrm{~K} 48$, and $\mathrm{K} 63$ ). All seven lysine residues of $\mathrm{Ub}$ can be conjugated one to other forming poly-Ub chains that will tag on substrates with different topologies ${ }^{5,6}$. The poly-Ub chains can constitute by a mixture of different lysine residues or by only one kind of lysine residue ${ }^{6}$, i.e. specific lysine-linked ploy-Ub chains (LLPUCs). The polyubiquitylation of substrates by different LLPUCs provides different pathways of their substrates ranging from proteolysis to non-proteolytic processes ${ }^{7-9}$. For example, LLPUCs K48 and K11 are considered for proteasomal degradation, whereas LLPUC K63 is involved in membrane protein endocytosis and autophagy ${ }^{6,10}$. LLPUCs of $\mathrm{K} 11, \mathrm{~K} 48$, and $\mathrm{K} 63$, have been identified to 
be related to F508del degradation ${ }^{6,11-13}$. However, the mechanism and details of ubiquitination of F508del are not fully clear yet. Understand of ubiquitination mediated F508del proteasomal degradation is necessary to facilitate the development of CF therapy and treatment in the future.

In this study, we examined all seven LLPUCs of Ub in F508del biogenesis. The data indicated that two of them, LLPUCs of K11 and K48, facilitated F508del degradation whereas the other two, LLPUCs of K63 and K33, prevented F508del from degradation. LLPUC of K11, instead of K48, is more potent for F508del degradation. Ub E3 ligase RNF5 was confirmed promoting LLPUCs of K48 and K11 formation and E3 ligase RNF5 exclusively catalyzed LLPUC of K11. Consequently, F508del modified by different LLPUCs is differentially recognized by the proteasomal shuttle protein and proteasomal receptors. These findings render new insights onto F508del degradation and a mechanistic explanation of diverse fates of different LLPUCs conjugated F508del.

\section{Results}

\section{Differential regulation of F508del degradation by different LLPUCs}

To investigate LLPUCs' function on F508del, we produced 17 ubiquitin mutants (Supplementary Figure S1A). The wild-type (WT) ubiquitin molecule contains seven lysine residues (K) that are key to the formation of LLPUCs. In the first group of mutants, we kept only one lysine residue and changed all the rest six lysine residues to arginine (R), namely $\mathrm{K} 6, \mathrm{~K} 11, \mathrm{~K} 27, \mathrm{~K} 29, \mathrm{~K} 33, \mathrm{~K} 48$, and $\mathrm{K} 63$ where the number indicates the kept lysine residue position. In the second group of mutants, only one lysine at the specific positions was mutant to arginine, namely K6R, K11R, K27R, K29R, K33R, K48R and K63R. In mutant K0, all seven lysines were changed to $R$.

We first looked at the expression levels of F508del in HEK293 cells cotransfected with F508del and WT $\mathrm{Ub}$ or the mutants. As expected, in mutant $\mathrm{KO}$ where there are no lysine residues left which consequently cannot ubiquitinate F508del, the F508del expression level increased by approximately 3.4 fold. In mutants that had only one lysine residue left, K6, K27 and K29 had no obvious effects on F508del expressions, whereas mutants K11 or K48 led to lower F508del expression although the effect sizes are moderate and not statistically significant compared to $\mathrm{Ub}$. The remaining two mutants, $\mathrm{K} 33$ or $\mathrm{K} 63$, had opposite effects, lead to 4-6 folds increase of F508del expressions (Fig. 1A \& B).

To further investigate the roles of $\mathrm{K} 33$ and $\mathrm{K} 63$, we made mutant $2 \mathrm{~K}$ in which the two lysine residues at positions $\mathrm{K} 33$ and $\mathrm{K} 63$ were kept while the other five lysine residues were mutated to arginine; conversely, we made mutant $2 \mathrm{KO}$ which carries arginine at position of $\mathrm{K} 33$ and $\mathrm{K} 63$ while the other five remain lysine residues (Supplementary Figure S1A). We looked at mutants 2K and 2K0 on their effects on F508del expression in HEK293 cells. By mutant 2K, F508del expression was significantly increased approximately 7.4 fold; consistently, mutant $2 \mathrm{KO}$ showed by dramatically decreasing the F508del expression to near zero 
(Fig. 1C \& D). These data suggested that LLPUCs K33 and K63, alone or in synergy, prevent F508del from degradation.

When these Ub mutants were co-transfected with WT-CFTR to HEK293 cells, the effects on CFTR mature form ( $C$ band) expression were minor than on immature form (B band) (Supplementary Figure S1B \& C), indicating that LLPUCs affected the immature CFTR in ER more than mature CFTR, consistent with prior reports ${ }^{14,15}$.

Science K33 and K63 increased and K11 and K48 decreased expressions of F508del (Figs. 1 and 2), we incubated F508del and Ub mutants transfected HEK293 cells with $3 \mu \mathrm{M}$ CFTR corrector VX-809 for 24 hours. Here we show that mature band was increased approximately 1.3 fold in cells with K33 and K63 than with Ub, while K11 and K48 mutants decreased their expressions (Fig. 1E \& F). Consistently, mutants $2 \mathrm{~K}$ or $2 \mathrm{KO}$ led to expected synergistic increase or decrease of F508del expressions at low temperature $\left(27^{\circ} \mathrm{C}\right)$ (Supplementary Figure S1D \& E). These results indicate that modulating these lysine residuals is compatible with CFTR correctors.

Next, to show F508del is conjugated by these LLPUCs naturally, we performed CFTR immunoprecipitation (IP) using CFTR antibody from cell lysates of CFBE-F508del, a human bronchial epithelial cell line with stable overexpression of F508del ${ }^{16}$. It's confirmed that F508del interacted with endogenous LLPUCs of K11, K48, and K63 (Fig. 1G, no commercial K33 antibody). It's confirmed that specific LLPUCs tagged F508del is naturally.

To gain more insight into the roles of LLPUCs by K11, K48, K33 and K63, the metabolic stability of F508del was examined using cycloheximide (CHX) chase. HEK293 cells co-transfected with F508del and Ub mutants were collected at designated time points $(0,30,60,90$ and $120 \mathrm{~min})$ to determine the expression levels and the half-life of F508del. We show that LLPUCs of K11 and K48 promoted F508del degradation, which was reversed by their mutations K11R and K48R (Fig. 2A-C \& 2G). In contrast, LLPUCs of K33 and K63 prolonged half-life of F508del, which was reversed by K33R or K63R (Fig. 2D, 2E, 2G-2I). The half-life of F508del is less than 45 minutes in our study (Fig. 2A, 2G, 2H \& 2I), consistent with the previous report ${ }^{16,17}$. Encouragingly, the half-life of F508del extended to approximately 6 hours by K33, $\mathrm{K} 63$, or $2 \mathrm{~K}$ (Fig. $2 \mathrm{G} \& 2 \mathrm{H}$ ). No obvious change of the half-life of F508del was detected induced by K27 and its mutant K27R (Fig. 2F, 2G). These findings were confirmed in CFBE-F508del cells (Supplementary Figure S2).

Taken together, these results demonstrated that LLPUCs of K11 and K48 promoted F508del degradation; whereas LLPUCs of K33 and K63 protected F508del from degradation.

\section{K33 and K63 promoted F508del forming aggregates}

We next employed confocal immunofluorescence microscopy to determine the cellular distribution of F508del in the presence of different Ub mutants. GFP-F508del and HA tagged Ub mutations were 
transfected into HEK293 cells and subjected to immunostaining. In cells with Ub mutants (i.e. K11 and K48) that had adverse effects in F508del expression levels, F508del mainly located in the perinuclear regions, similar to those co-transfected with WT-Ub or vector (Fig. 3A). In cells co-transfected with Ub mutants that could enhance F508del expression levels (i.e. K0, K33 or K63), GFP-F508del formed aggregate-like bodies that were co-localized with Ub mutants in approximately $50 \%$ of the cells with cooverexpressing of $\mathrm{K} 33, \mathrm{~K} 63$ or $\mathrm{KO}$ and $\mathrm{F} 508 \mathrm{del}$ (Fig. $3 \mathrm{~A} \& \mathrm{~B}$ ), in contrast to less than $10 \%$ of cells with GFP-F508del aggregates of those co-transfected with F508del and Ub, K11, or K48 (Fig. 3A \& B, n= 50, P $<0.001$ ). Moreover, in cells with granules the brightness of fluorescence, an indicator of the quantity of GFP-F508del aggregates, was much stronger in cells expressing K0, 33 or 63 than those with Ub, K11, or K48 (Fig. 3C, $n=50, P<0.001$ ).

We also separated the soluble and insoluble fragments from HEK293 cells co-transfected with F508del and different lysine mutants. With K33 and K63 overexpressing, F508del dramatically increased in both soluble and insoluble fractions compared to that of WT-Ub overexpression, (3.7 vs. 4.4 and 2.6 vs. 3.6, Supplementary Figure S3); whereas low amount of F508del was found in both soluble and insoluble forms from cell with Ub, K11 and K48 overexpression (Supplementary Figure S3). These data further confirmed that $\mathrm{Ub} \mathrm{K} 33$ and $\mathrm{K} 63$ mutants prevented F508del from degradation and indicated that these $\mathrm{Ub}$ mutants promote F508del forming aggregates.

\section{Ubiquitination of F508del did not elicit does not elicit additional ER stress or Proteasome activity}

To elucidate the potential mechanism of how LLPUCs regulate F508del biogenesis, we firstly analyzed the endoplasmic reticulum (ER) stress using real-time PCR and immunoblot in HEK 293 cells cotransfected with F508del and different Ub mutants. No significant change of ER stress markers were observed when F508del and Ub mutants were overexpressed, regardless of the mutation loci (Fig. 4A - C). In addition, we analyzed and the proteasome activity which increased slightly in cells co-expressing K33 or K63 (Fig. 4D), however without achieving statistical significance $(n=3, p>0.05)$.

\section{E3 Ligases involved in LLPUC formation on F508del}

E3 ligases are highly specific on ubiquitinylation of their target substrates ${ }^{18}$. It has been reported that two ubiquitin E3 ligases, RNF5 (also known as RMA1) and CHIP, drive the degradation of F508del sequentially in its early biogenesis ${ }^{19}$. To check which of the LLPUCs are catalyzed by RNF5 and CHIP during F508del biogenesis, we examined the involvement of these E3s in LLPUCs conjugation on F508del by transfecting HEK293 cells with plasmids expressing E3 ligases, Ub and/or their mutants (Fig. 5).

Both RNF5 and CHIP accelerated F508del degradation when co-transfected with K48, which was reversed by K48R. Consistently, the dominant negative mutations of RNF5 and CHIP, RNF5CS and CHIPdUb respectively, could not degrade K48 conjugated F508del; whereas F508del degradation was diminished 
by overexpression of dominant negative forms of these two E3s or K48R, especially by the synergy of E3 mutants and K48R (Fig. 5). Interestingly, F508del degradation was also promoted by co-expression of RNF5 and K11 by the similar way, but not CHIP, because the level of F508del expression was similar between K11 and K11R co-transfected with CHIP or CHIPdUb (Fig. 5).

These results strongly suggested that RNF5 and CHIP were both involved in K48 LLPUC formation and that RNF5 but not CHIP catalyzed K11 LLPUC formation on F508del.

\section{Differential physical interaction of LLPUCs ubiquitylated F508del with the proteasomal shuttle and receptors.}

During the degradation process, the ubiquitinated proteins are transported to the proteasome by proteasomal shuttle proteins, and then recognized by ubiquitin receptors on $26 \mathrm{~S}$ proteasome ${ }^{20-22}$. Here we checked the interaction of proteasomal shuttle protein $(\operatorname{Rad} 23 \mathrm{~A})$, and proteasomal receptors (S5a and Adrm1) with different LLPUCs conjugated F508del in HEK293 cells co-transfected with F508del and different Ub lysine mutants for 48 hours and then treated with the proteasome inhibitor MG-132 for 4 hours. F508del was pulled down by indicated GST-fusion proteins of those shuttle or receptors (Supplementary Figure S4) from cell lysates with the same amount of ubiquitilyzed F508del and detected using immunoblot (Fig. 6). The interaction between Rad23A and F508del appears to be similar between the control group and the cells co-transfected with Ub K48 mutant. Such interaction appears to be weaker in cells co-transfected with $\mathrm{K} 11, \mathrm{~K} 33$ or $\mathrm{K} 63$ mutants, suggesting that Rad23A is specific in transferring LLPUC of K48 conjugated F508del (Fig. 6A \& E).

The interaction between the proteasomal receptor S5a and F508del was attenuated in cells cotransfected with $\mathrm{K} 33$ or $\mathrm{K} 63$, but enhanced in cells co-transfected with $\mathrm{K} 11$ or $\mathrm{K} 48$, in comparison to the control group (Fig. 6B \& E). Similarly, the interaction between Adrm1 and F508del was weakened in K33 and K63 groups, but enhanced in the K11 group (Fig. 6C \& E).

These data showed that Rad23A and S5a facilitated F508del degradation by recognizing LLPUC of K48, while S5a and Adrm1 promoted F508del degradation by recognizing LLPUC of K11. Based on these findings, we propose that the fate of F508del is at least partially decided by the affinity between the proteasome shuttle/receptors with differential LLPUCs, i.e. the affinity was enhanced by proteolytic related LLPUC tags, such as K11 and K48, nevertheless, which became weak by no-proteolytic LLPUCs of $\mathrm{K} 33$ and $\mathrm{K} 63$.

\section{Discussion}

F508del is a typical substrate degraded by the proteasome, but the process is not fully known to date 15,23 . To gain further mechanistic insights of Ub-mediated F508del degradation, we examined all seven lysine residues forming poly-Ub chains in F508del biogenesis and demonstrated that four LLPUCs were 
involved in F508del degradation regulation, i.e. LLPUCs of K11 and K48 facilitated F508del proteasomal degradation, where LLPUCs of K33 and K63 mediated F508del from degradation.

LLPUCs conjugation of CFTR is associated with various biochemical processes. For example, LLPUCs of $\mathrm{K} 11$ and $\mathrm{K} 48$ are related to $\mathrm{F} 508$ del proteasomal degradation ${ }^{13}$, and $\mathrm{K} 63$-linked ubiquitin chain acts as a non-proteolytic role, including CFTR endocytosis, lysosomal degradation, and autophagy 10,24-26. However, the details of how these LLPUCs regulate F508del remain to be fully elucidated ${ }^{24,27}$.

$\mathrm{K} 11$ and $\mathrm{K} 48$ conjugated F508del is known to suffer from a quick degradation by the proteasome ${ }^{13}$. K48 ubiquitylation correlates the recruitment of proteasomal subunits ${ }^{10}$. Like K48, LLPUC of K11 conjugated substrates are also targeted to proteasomal degradation ${ }^{21,28,29}$. In fact, the so-called "non-canonical" $\mathrm{K} 11$ linkage is as abundant as the "canonical" K48 linkages in cells ${ }^{13,30}$. Consistently, our findings show that both LLPUCs of K11 and K48 facilitated F508del degradation, and that K11 ubiquitinylation facilitates F508del degradation more than K48, in line with an early report by Dr. Rape's group that the anaphase-promoting complex (APC/C) is degraded more when conjugated by $\mathrm{K} 11$ rather than by $\mathrm{K} 48$ 29,31 . Collectively, the present work and early reports suggest that $\mathrm{K} 11$ is more potent than $\mathrm{K} 48$ for F508del degradation.

In this study, we found that K33 or K63 overexpression had rescue effects in F508del degradation. The half-life of F508del is usually 30 to 45 minutes ${ }^{16,17}$, whereas we identified that K33 or K63 prolonged F508del's half-life to approximately 6 hours, an effect that was reversed by K33R or K63R.

However, our results are conflictive with K63's aggresome clearance function that has been previously reported ${ }^{10,25,32-34}$, as we observed that the F508del expression level increased dramatically by K63 overexpression and F508del even formed aggregate-like granules. On the other hand, several other groups recently reported similar roles of Ub K63 to ours. Misfolded F508del is an aggresome-prone protein that results in autophagy defective in CF patients ${ }^{35}$. In the latest report by Bellia et al., the UPS activity is reduced in Alzheimer's Disease patients, which is induced by amyloid $\beta$ peptide that binding to ubiquitin by residues $\mathrm{K}_{63}{ }^{36}$. In the other work by Jena et al. it is revealed that TRIM16 activates NFE2L2/NRF2 by K63-linked ubiquitination that upregulates the $\mathrm{p} 62$ and results in misfolding protein aggregate formation 37 . Thus, we speculated that LLPUC of K63 binding F508del, at least in our system, favors aggregates formation. Interestingly, co-transfecting K63 enhanced the efficacy of CFTR corrector VX-809 in HEK293 cells, suggesting this pathway's potential value in CF therapy.

The present work provided novel knowledge to the functions of LLPUC of K33, which is still largely unknown ${ }^{27,38,39}$. LLPUC of K33 has also been reported conjugating p62 for aggresome formation ${ }^{27,40}$. Our data suggested that $\mathrm{K} 33$ possibly shares a similar function with $\mathrm{K} 63$, as these two $\mathrm{Ub}$ mutants led to similar effects in our experiments. Further research is necessary to elucidate the details for K33 in F508del regulation. 
E3 ligase RNF5 and CHIP are known as key players regulating F508del proteasomal degradation ${ }^{19}$. We found that RNF5 synthesized both LLPUCs of K11 and K48 formation, CHIP facilitated LLPUC of K11 (but not K48) formation, and that neither of them catalyzed LLPUCs of K33 or K63. Interestingly while usually one E3 ligase was considered to synthesize only one type of ubiquitin chain ${ }^{41}$, our data suggested that RNF5 catalyzed both of LLPUCs of K11 and K48 formation.

In this study, we analyzed the interaction between four LLPUCs modified F508del and the proteasomal shuttle protein (Rad23a) or the proteasomal receptors (S5a and Adrm1). R23a, with C- terminal ubiquitinassociated (UBA) domain, preferentially binds K48-linked chains of substrates and transfer substrates to the proteasome ${ }^{42}$. S5a and Adrm1 on the 26S proteasome prefer to recognize K48 and K11 linkage ubiquitinylated substrates for degradation ${ }^{21,28}$. Our data were similar to those in a most recently published report that S5a preferably selects K48- or K11- linked substrates ${ }^{21}$. We hence hypothesize that differential LLPUCs ubiquitylation alter the affinity between F508del and its proteasomal shuttle and/or receptors.

\section{Conclusion}

In the present research, we found F508del utilizes four specific lysine-linked poly Ub chains and during its biogenesis: LLPUCs of K11 and K48 promotes F508del proteasomal degradation; but LLPUCs of K33 and K63 conjugated F508del escaped degradation likely due to failed recognized by proteasomal shuttle protein or receptors. These findings provide new insights to understand the CF pathogenesis and are expected to facilitate the development of therapies for this devastating disease.

\section{Methods}

\section{Antibodies and Chemicals}

CFTR antibody 217 was purchased from the University of North Carolina at Chapel Hill. Antibodies targeting HA and Myc epitope, BIP, CHOP, PERK, CNX, PDI, P-elf2a, elf2a, ubiquitin, and K63, K48, K33, and actin antibodies were purchased from Cell Signaling. HRP-secondary antibodies were from Abcam. F508del in the pcDNA3.1 vector was described previously ${ }^{43}$. The N-terminal HA-tagged ubiquitin was amplified from human cDNA (Invitrogen) and subcloned into pRK5, which was used as a template for other $\mathrm{Ub}$ mutants for different lysine mutations using the site-directed mutagenesis kit (Stratagene). pcDNA3.1-FLAG-RNF5, pcDNA3.1-FLAG-RNF5CS were from Dr. Cyr ${ }^{19}$, pGEX6P-GST-Rad23a, pGEX6PGST-Adrm1, and pGEX6P-GST-S5a were from Addgene. All plasmids were confirmed by DNA sequencing. MG-132, Cycloheximide, IPTG, and tunicamycin were from Sigma. Glutathione Sepharose 4B was from Fisher Scientific. TRIzol reagent was purchased from Invitrogen.

\section{Cell culture and transfection}


HEK293 cells were cultured in Dulbecco's modified Eagle's (DMEM, Invitrogen) supplemented with $10 \%$ fetal bovine serum. CFBE-F508del cell was cultured in MEM (Gibco) with 10\% fetal bovine serum and 1 $\mu \mathrm{g} / \mathrm{ml}$ puromycin. All cells were maintained in a humidified atmosphere containing $5 \% \mathrm{CO}_{2}$ at $37^{\circ} \mathrm{C}$. Cells were transiently transfected using Lipofectamine 3000 (Invitrogen) and harvested at 48 hours posttransfection.

\section{Cycloheximide (CHX) chase}

About 44 hours after transfection, cells were incubated with $20 \mu \mathrm{g} / \mathrm{ml} \mathrm{CHX}$ in fresh full medium and were harvested at identified times points.

\section{GST pull-down}

pGEX6P plasmids were transformed into E. coli BL21(DE3) cells. After confirmation, the bacteria were induced with $100 \mu \mathrm{M}$ IPTG and cultured for an additional 4.5 hours when the OD of bacteria was reached 0.4. Then the bacteria were collected and lysed, of which the supernatant was used as the total protein. GST fusion proteins were purified from the supernatant using Glutathione Agarose Resin (Pierce), which were analyzed by SDS-PAGE and stained with $0.25 \%$ Coomassie Blue R-250.

\section{Quantitative real-time reverse transcription PCR analysis}

Total cellular RNA was extracted using TRIzol reagent and reverse-transcribed to cDNA using a random primer. The real-time PCR reaction mixture containing CDNA template, primers and SYBR Green PCR Master Mix (Invitrogen) was run in a 7,500 Fast Real-time PCR System (Applied Biosystems). Fold changes of mRNA levels were determined after normalization to internal control of $\beta$-actin RNA levels ${ }^{44}$.

\section{The activity of 20S proteasome core}

HEK293 cells transfected with indicated plasmids for 48 hours. $10 \mu \mathrm{g}$ total protein from each sample was subjected to proteasome activity assay in which an AMC-tagged peptide substrate was added, and the free and highly fluorescent $A M C$ was released in the presence of proteasome activity. After incubation at $37^{\circ} \mathrm{C}$ for $60 \mathrm{~min}$, fluorescence was then measured by microplate reader with $\mathrm{Ex} / \mathrm{Em}=350 / 440 \mathrm{~nm}$.

\section{Soluble and insoluble fractions extraction}

Lysed cells by homogenization in lysis buffer $(120 \mathrm{mM} \mathrm{NaCl}, 50 \mathrm{mM}$ Tris, with protease inhibitors, $\mathrm{pH}$ 8.0 ) and centrifuged the lysate at $15,000 \times \mathrm{g}$ for $30 \mathrm{~min}$ at $4{ }^{\circ} \mathrm{C}$. The supernatant is soluble fraction and the pellet is insoluble fraction. 
Statistical analyses. Statistical analyses were performed using the unpaired, 2-tailed Student's t-test. All quantitative data presented were from three independent experiments and were shown as the mean \pm SD. Statistical tests reached $p<0.05$ were considered the statistical significance.

\section{Abbreviations}

CF: cystic fibrosis; CFTR: cystic fibrosis transmembrane conductance regulator; wild-type CFTR: WT-CFTR; F508del-CFTR: F508del; Ub: ubiquitin; ER: endoplasmic reticulum; ERAD: ER associated protein degradation; UPS: ubiquitin proteasome system; LLPUC: lysine-linked polyubiquitin chains; UBA: ubiquitin binding-Associated.

\section{Declarations}

\section{Ethics approval and consent to participate}

Not applicable.

\section{Consent for publication}

Not applicable.

\section{Competing of interests}

The authors declare no competing interests.

\section{Funding}

This work was supported by National Science Foundation of China (31400651), Natural Science Foundation of Heilongjiang Province, China (LH2020H003), Funding of Basic Medicine Team of Jiamusi University, China (JDXKTD-2019002), and Beiyao general Pharmaceutical Corporation,China.

\section{Author contributions}

QW, YH, GZ and XH were responsible for designed the experiments; QW and XH produced plasmids. QW, $\mathrm{YH}, \mathrm{XM}, \mathrm{LL}, \mathrm{XL}, \mathrm{NV}$, and $\mathrm{PZ}$ performed the cell and molecular biological experiments; $\mathrm{QW}$ and $\mathrm{XL}$ contributed the biotinylation experiments; GZ did the LC-MS/MS analysis. QW, GZ, YH, NV, and XH wrote the paper. All authors discussed the results and commented on the manuscript.

\section{Acknowledgements}


We thank all people shown in the paper and the people in their labs; specifically, we would like to acknowledge the Life Science Central Lab of Jiamusi University.

\section{References}

1. Pilewski JM, Frizzell RA. Role of CFTR in airway disease. Physiol Rev. 1999;79:215-55. doi:10.1152/physrev.1999.79.1.S215.

2. Knapp EA, et al. The Cystic Fibrosis Foundation Patient Registry. Design and Methods of a National Observational Disease Registry. Ann Am Thorac Soc. 2016;13:1173-9. doi:10.1513/AnnalsATS.201511-7810C.

3. Amaral MD. CFTR and chaperones: processing and degradation. J Mol Neurosci. 2004;23:41-8. doi:10.1385/JMN:23:1-2. 041.

4. Zhang Z, Baksh MM, Finn MG, Heidary DK, Richards Cl. Direct Measurement of Trafficking of the Cystic Fibrosis Transmembrane Conductance Regulator to the Cell Surface and Binding to a Chemical Chaperone. Biochemistry. 2017;56:240-9. doi:10.1021/acs.biochem.6b00853.

5. Song L, Luo ZQ. Post-translational regulation of ubiquitin signaling. J Cell Biol. 2019. doi:10.1083/jcb.201902074.

6. Li W, Ye Y. Polyubiquitin chains: functions, structures, and mechanisms. Cell Mol Life Sci. 2008;65:2397-406. doi:10.1007/s00018-008-8090-6.

7. Christianson JC, Ye Y. Cleaning up in the endoplasmic reticulum: ubiquitin in charge. Nat Struct Mol Biol. 2014;21:325-35. doi:10.1038/nsmb.2793.

8. Komander D, Rape M. The ubiquitin code. Annu Rev Biochem. 2012;81:203-29. doi:10.1146/annurev-biochem-060310-170328.

9. Shaid S, Brandts $\mathrm{CH}$, Serve H, Dikic I. Ubiquitination and selective autophagy. Cell Death Differ. 2013;20:21-30. doi:10.1038/cdd.2012.72.

10. Erpapazoglou Z, Walker O, Haguenauer-Tsapis R. Versatile roles of k63-linked ubiquitin chains in trafficking. Cells. 2014;3:1027-88. doi:10.3390/cells3041027.

11. McClure ML, Barnes S, Brodsky JL, Sorscher EJ. Trafficking and function of the cystic fibrosis transmembrane conductance regulator: a complex network of posttranslational modifications. Am J Physiol Lung Cell Mol Physiol. 2016;311:L719-33. doi:10.1152/ajplung.00431.2015.

12. Michel MA, et al. Assembly and specific recognition of k29- and k33-linked polyubiquitin. Mol Cell. 2015;58:95-109. doi:10.1016/j.molcel.2015.01.042.

13. Xu P, et al. Quantitative proteomics reveals the function of unconventional ubiquitin chains in proteasomal degradation. Cell. 2009;137:133-45. doi:10.1016/j.cell.2009.01.041.

14. Ward CL, Omura S, Kopito RR. Degradation of CFTR by the ubiquitin-proteasome pathway. Cell. 1995;83:121-7. doi:10.1016/0092-8674(95)90240-6.

15. Jensen TJ, et al. Multiple proteolytic systems, including the proteasome, contribute to CFTR processing. Cell. 1995;83:129-35. doi:10.1016/0092-8674(95)90241-4. 
16. Hou X, et al. Dissection of the Role of VIMP in Endoplasmic Reticulum-Associated Degradation of CFTRDeltaF508. Sci Rep. 2018;8:4764. doi:10.1038/s41598-018-23284-8.

17. Varga $\mathrm{K}$, et al. Efficient intracellular processing of the endogenous cystic fibrosis transmembrane conductance regulator in epithelial cell lines. J Biol Chem. 2004;279:22578-84. doi:10.1074/jbc.M401522200.

18. Hegde RN, et al. Unravelling druggable signalling networks that control F508del-CFTR proteostasis. Elife 4, doi:10.7554/eLife.10365 (2015).

19. Younger JM, et al. Sequential quality-control checkpoints triage misfolded cystic fibrosis transmembrane conductance regulator. Cell. 2006;126:571-82. doi:10.1016/j.cell.2006.06.041.

20. Sutton JR, et al. Interaction of the polyglutamine protein ataxin-3 with Rad23 regulates toxicity in Drosophila models of Spinocerebellar Ataxia Type 3. Hum Mol Genet. 2017;26:1419-31. doi:10.1093/hmg/ddx039.

21. Chen X, et al. Structure of hRpn10 Bound to UBQLN2 UBL Illustrates Basis for Complementarity between Shuttle Factors and Substrates at the Proteasome. J Mol Biol. 2019;431:939-55. doi:10.1016/j.jmb.2019.01.021.

22. Chen X, et al. Structures of Rpn1 T1:Rad23 and hRpn13:hPLIC2 Reveal Distinct Binding Mechanisms between Substrate Receptors and Shuttle Factors of the Proteasome. Structure. 2016;24:1257-70. doi:10.1016/j.str.2016.05.018.

23. Ramachandran S, et al. NEDD8, and FBXO2 Proteins Regulate DeltaF508 Cystic Fibrosis Transmembrane Conductance Regulator (CFTR) Ubiquitin-mediated Proteasomal Degradation. J Biol Chem 291. 2016;SYVN1:25489-504. doi:10.1074/jbc.M116.754283.

24. Fushman D, Walker O. Exploring the linkage dependence of polyubiquitin conformations using molecular modeling. J Mol Biol. 2010;395:803-14. doi:10.1016/j.jmb.2009.10.039.

25. Okiyoneda T, et al. Chaperone-Independent Peripheral Quality Control of CFTR by RFFL E3 Ligase. Dev Cel/ 44, 694-708 e697, doi:10.1016/j.devcel.2018.02.001 (2018).

26. Yazlovitskaya EM, et al. The laminin binding alpha3 and alpha6 integrins cooperate to promote epithelial cell adhesion and growth. Matrix Biol. 2019;77:101-16. doi:10.1016/j.matbio.2018.08.010.

27. Nibe $Y$, et al. Novel polyubiquitin imaging system, PolyUb-FC, reveals that K33-linked polyubiquitin is recruited by SQSTM1/p62. Autophagy 14, 347-358, doi:10.1080/15548627.2017.1407889 (2018).

28. Bonacci T, Emanuele MJ. Impressionist portraits of mitotic exit: APC/C, K11-linked ubiquitin chains and Cezanne. Cell Cycle. 2019;18:652-60. doi:10.1080/15384101.2019.1593646.

29. Meyer HJ, Rape M. Enhanced protein degradation by branched ubiquitin chains. Cell. 2014;157:91021. doi:10.1016/j.cell.2014.03.037.

30. Castaneda CA, Kashyap TR, Nakasone MA, Krueger S, Fushman D. Unique structural, dynamical, and functional properties of k11-linked polyubiquitin chains. Structure. 2013;21:1168-81. doi:10.1016/j.str.2013.04.029. 
31. Jin L, Williamson A, Banerjee S, Philipp I, Rape M. Mechanism of ubiquitin-chain formation by the human anaphase-promoting complex. Cell. 2008;133:653-65. doi:10.1016/j.cell.2008.04.012.

32. Garcia-Mata R, Gao YS, Sztul E. Hassles with taking out the garbage: aggravating aggresomes. Traffic. 2002;3:388-96.

33. Kamsteeg EJ, et al. Short-chain ubiquitination mediates the regulated endocytosis of the aquaporin-2 water channel. Proc Natl Acad Sci U S A. 2006;103:18344-9. doi:10.1073/pnas.0604073103.

34. Henry KR, Lee S, Walker D, Zeitlin PL. Direct interactions between ENaC gamma subunit and CICN2 in cystic fibrosis epithelial cells. Physiol Rep 3, doi:10.14814/phy2.12264 (2015).

35. Luciani A, et al. Defective CFTR induces aggresome formation and lung inflammation in cystic fibrosis through ROS-mediated autophagy inhibition. Nat Cell Biol. 2010;12:863-75. doi:10.1038/ncb2090.

36. Bellia F, et al. Ubiquitin binds the amyloid beta peptide and interferes with its clearance pathways. Chem Sci. 2019;10:2732-42. doi:10.1039/c8sc03394c.

37. Jena KK, et al. TRIM16 governs the biogenesis and disposal of stress-induced protein aggregates to evade cytotoxicity: implication for neurodegeneration and cancer. Autophagy. 2019;15:924-6. doi:10.1080/15548627.2019.1586251.

38. Kulathu Y, Komander D. Atypical ubiquitylation - the unexplored world of polyubiquitin beyond Lys48 and Lys63 linkages. Nat Rev Mol Cell Biol. 2012;13:508-23. doi:10.1038/nrm3394.

39. Dybas JM, et al. Integrative proteomics reveals an increase in non-degradative ubiquitylation in activated CD4(+) T cells. Nat Immunol. 2019;20:747-55. doi:10.1038/s41590-019-0381-6.

40. Heath RJ, et al. RNF166 Determines Recruitment of Adaptor Proteins during Antibacterial Autophagy. Cell Rep. 2016;17:2183-94. doi:10.1016/j.celrep.2016.11.005.

41. Qin Y, et al. RNF26 temporally regulates virus-triggered type I interferon induction by two distinct mechanisms. PLoS Pathog. 2014;10:e1004358. doi:10.1371/journal.ppat.1004358.

42. Chen L, Shinde U, Ortolan TG, Madura K. Ubiquitin-associated (UBA) domains in Rad23 bind ubiquitin and promote inhibition of multi-ubiquitin chain assembly. EMBO Rep. 2001;2:933-8. doi:10.1093/embo-reports/kve203.

43. Sun F, et al. Derlin-1 promotes the efficient degradation of the cystic fibrosis transmembrane conductance regulator (CFTR) and CFTR folding mutants. J Biol Chem. 2006;281:36856-63. doi:10.1074/jbc.M607085200.

44. Wang G, et al. Endoplasmic reticulum factor ERLIN2 regulates cytosolic lipid content in cancer cells. Biochem J. 2012;446:415-25. doi:10.1042/BJ20112050.

\section{Figures}


A

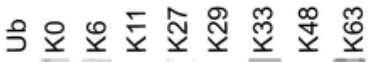

a-CFTR 0 a

(Ub)

a-Actin

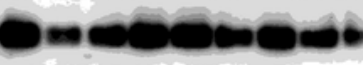

B

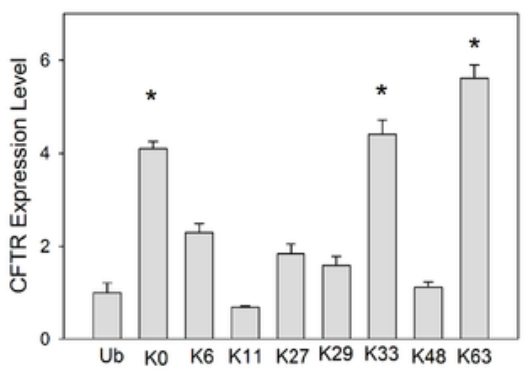

C

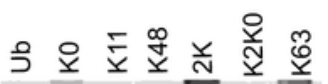

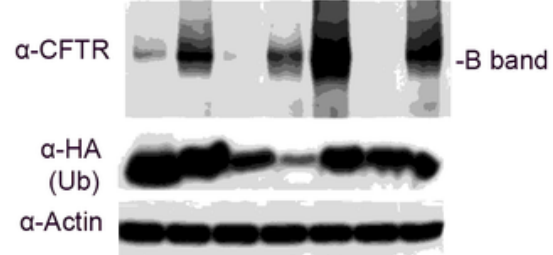

E
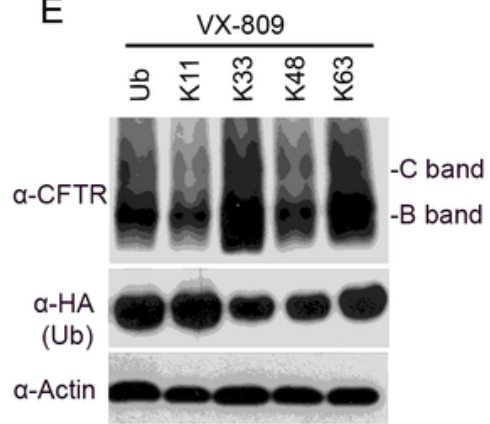

D

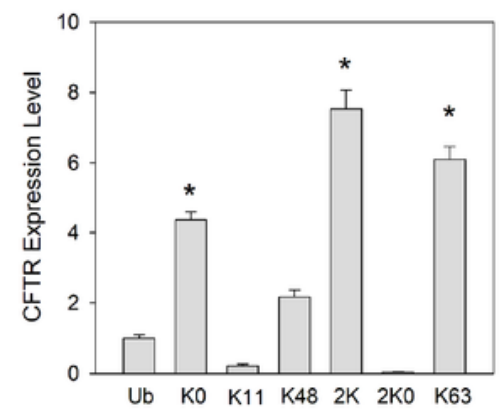

F

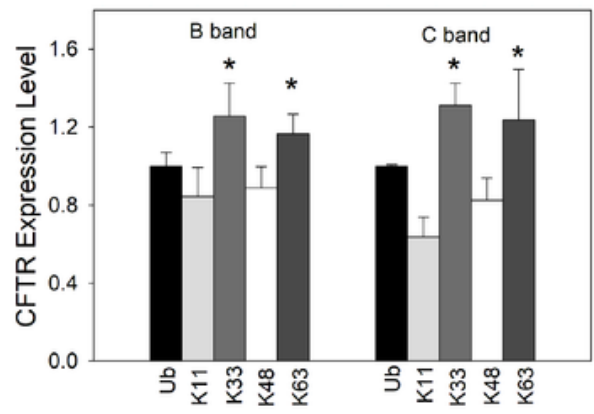

G

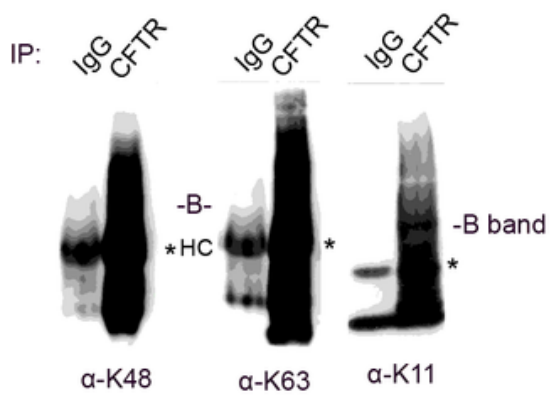

Figure 1

Differential regulation of F508del biogenesis by LLPUCs. A, C, HEK293 cells were co-transfected with indicated plasmids. $50 \mu \mathrm{g}$ total proteins from each transfection were subjected to immnuoblotting with indicated antibodies. B, D, Quantification of the CFTR signals in panel A and C (mean \pm SEM, $n=3$ ). Quantitation of F508del expressions (density) from each transfection is given below each lane after normalization to actin expression. E, HEK293 cells were transfected with indicated plasmids for 48 hours, 
incubated with $3 \mu \mathrm{M} V X-809$ for additional 24 hours, and then collected for western blotting. $F$, Quantification of the CFTR signals in panel $E$ (mean \pm SEM, $n=3$ ). G, $500 \mu$ g protein from HBE cell line CFBE-F508del was immunoprecipitated with CFTR antibody or IgG. Precipitates were then fractionated in SDS-PAGE gel and blotted with an antibody against K48, K63-linked, or K11. HC: antibody heavy chain.
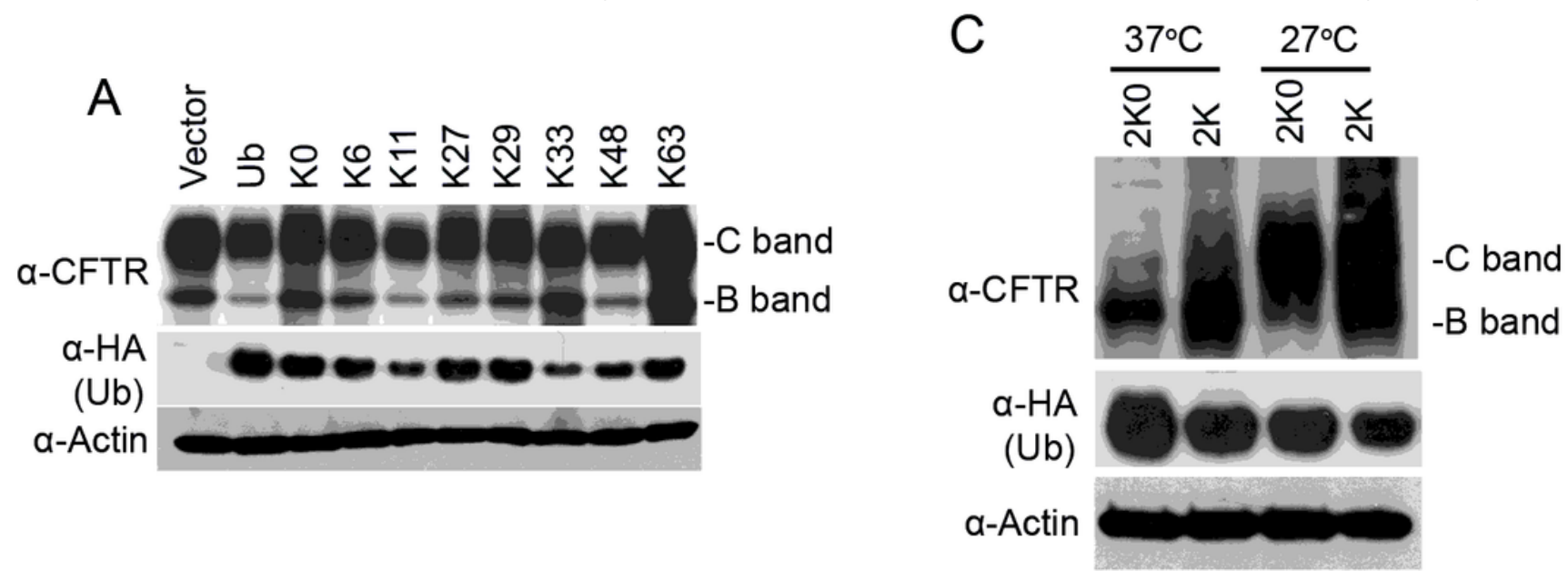

B

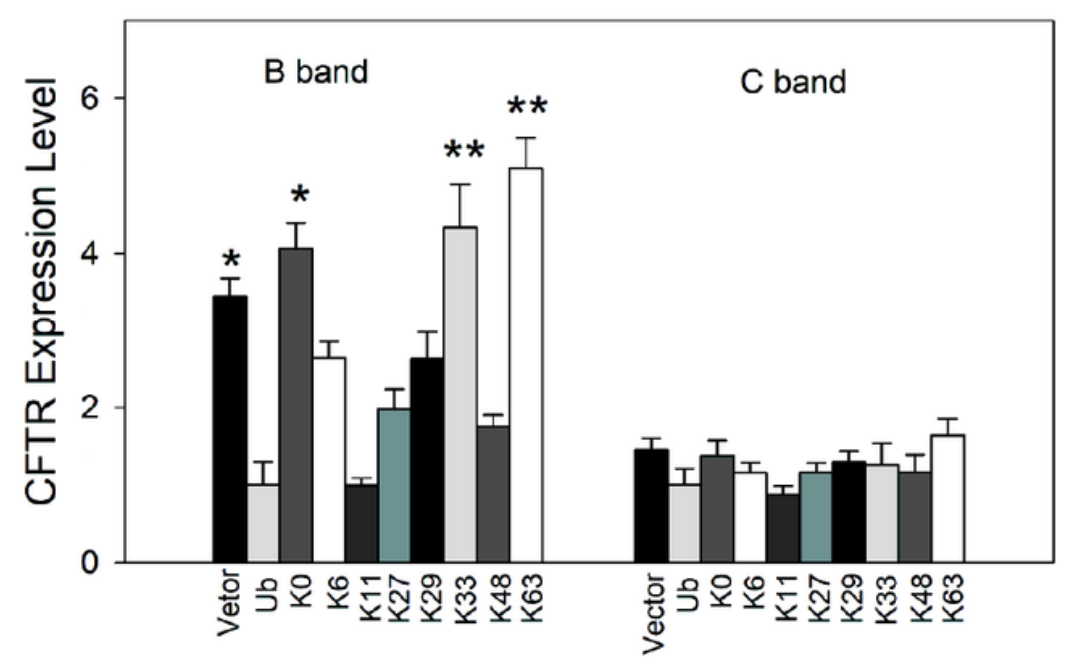

D

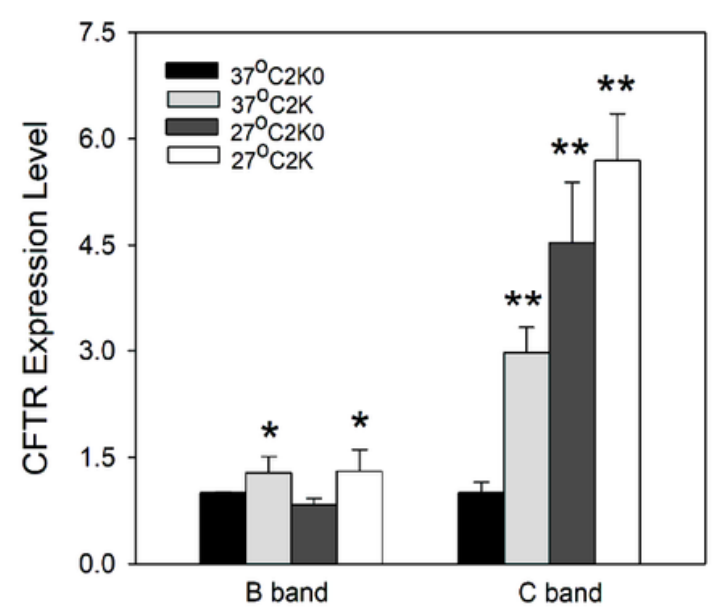

Figure 2

Dynamic effect of LLPUCs on F508del degradation. A-F, H, F508del was co-expressed with ubiquitin or its mutants in HEK293 cells. 48 hours post-transfection, and cells were treated with $50 \mu \mathrm{g} / \mathrm{ml}$ cycloheximide $(\mathrm{CHX})$ and harvested at different time points. Cell lysates were then subjected to immunoblotting with CFTR ab. G, I, quantitation of F508del signals of panel G from three experiments; values expressed as a percentage of band $B$ density at chase time $=0$. 
A

$\underset{(\min )}{\mathrm{CHX}} \frac{\mathrm{Ub}}{306090120} \frac{\mathrm{KO}}{306090120}$ $y=\Leftrightarrow \rightarrow-B$ band

D

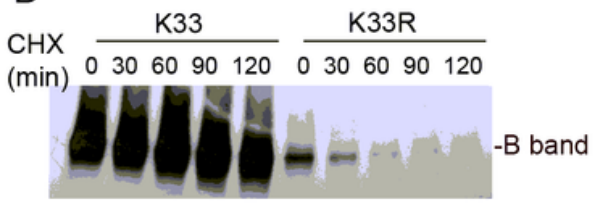

B

CHX $\frac{\mathrm{K} 11}{\min ) 0} \frac{\mathrm{K} 11 \mathrm{R}}{306090120} \frac{306090120}{030}$

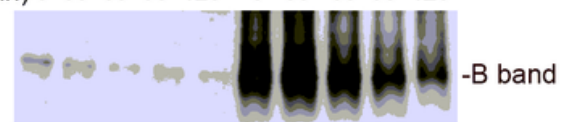

E

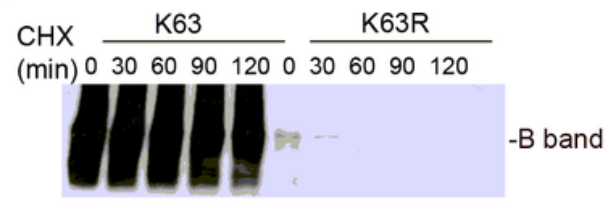

C

$\underset{(\min )}{\mathrm{CHX}} \frac{\mathrm{K} 48}{306090120} \frac{\mathrm{K} 48 \mathrm{R}}{306090120}$

-

F

$\underset{(\mathrm{min})}{\mathrm{CHX}} \frac{\mathrm{K} 27}{306090120} \frac{\mathrm{K} 27 \mathrm{R}}{0306090120}$
G

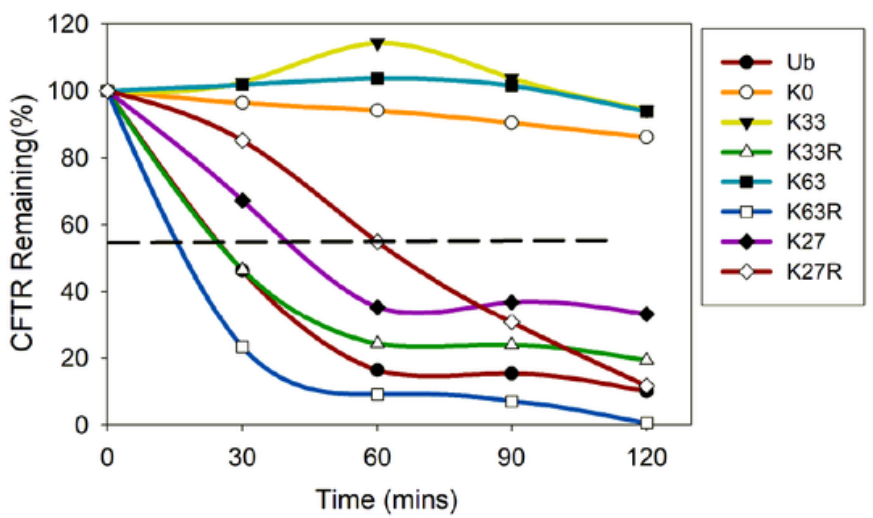

$\mathrm{H}$

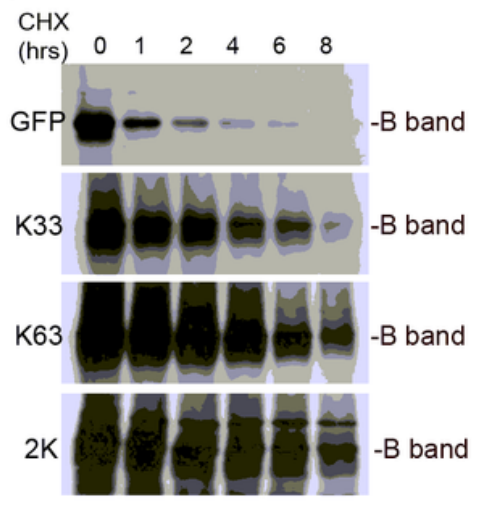

I

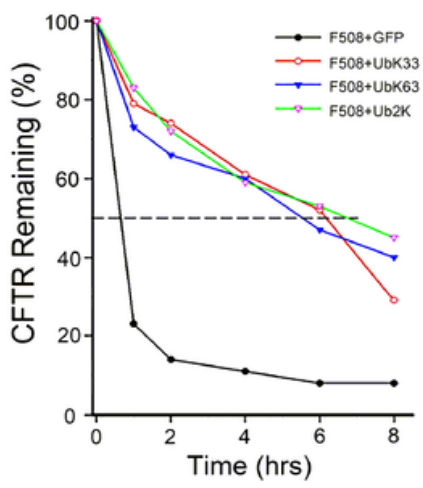

Figure 3

K33 and K63 conjugation induced F508del aggregation. A, HEK293 cells expressing GFP-F508del plus Ub or its mutants were subjected to confocal microscopy. The cells were stained with antibodies HA, and subsequently incubated with anti-mouse rhodamine labeled secondary antibodies. The cells were examined by confocal microscopy. Scale bar, $20 \mu \mathrm{m}$. B, C, Quantified cells in panel A with aggregates. (mean \pm SEM, $n=50$ ). 

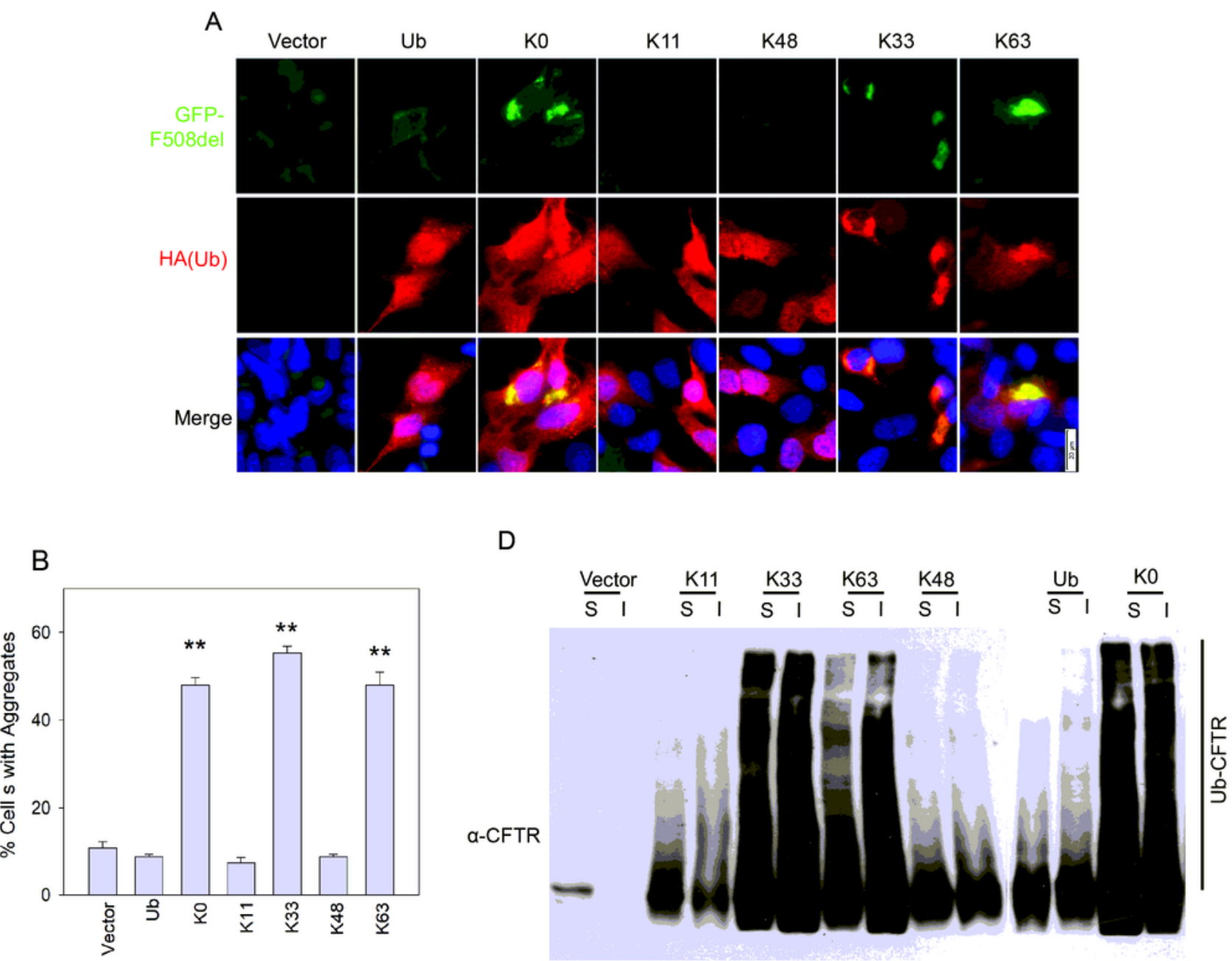

E
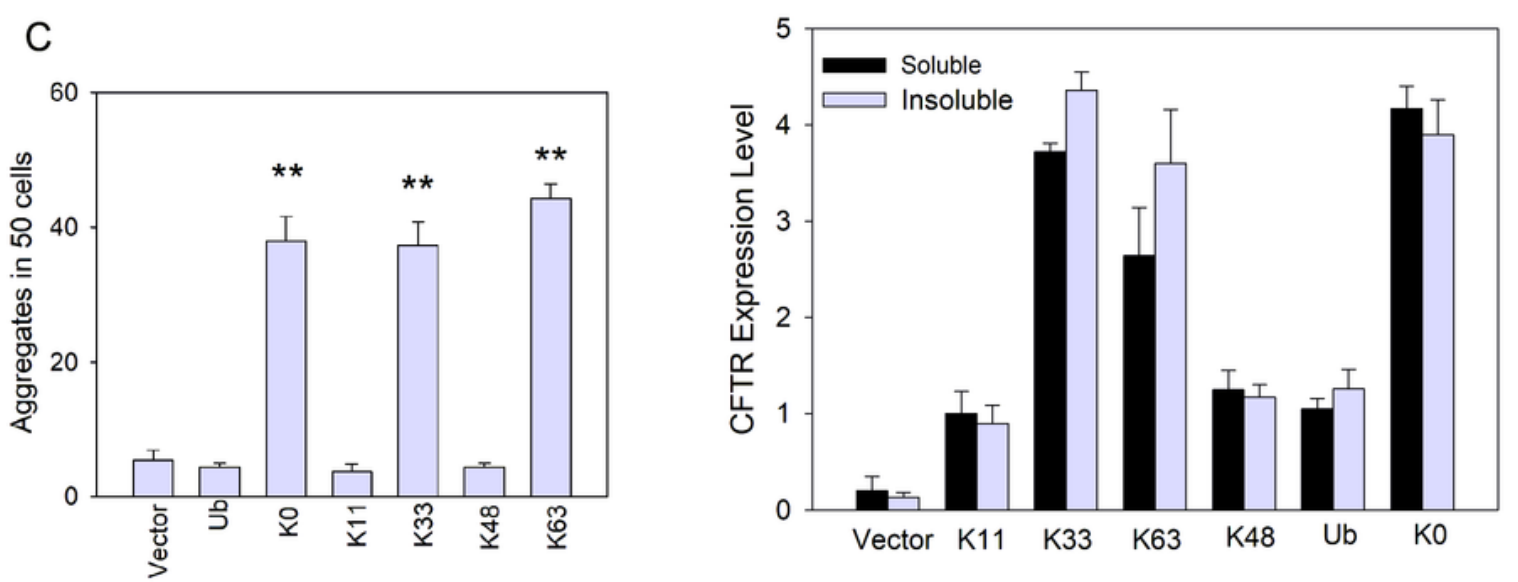

Figure 4

ER stress and Proteasome activity with Ubiquitination of F508del. HEK293 cells co-transfected with F508del and Ub or its mutants. Two-day posttransfection, total protein or RNAs from each sample were isolated and subjected to immunoblot or real-time PCR to check ER stress markers. A, ER stress markers were detected by Western blotting. B, Quantitation of proteins of panel A (mean $\pm S E, n=3$ ). C, ER stress markers were detected by real-time PCR and quantitated (mean $\pm S E, n=3$ ). $D$, The catalytic activity of 
20S proteasome core. Activity of proteasome in HEK293 cells transfected with indicated plasmids for 48 hours. Results are from three independent experiments (mean $\pm S E, n=3$ ).

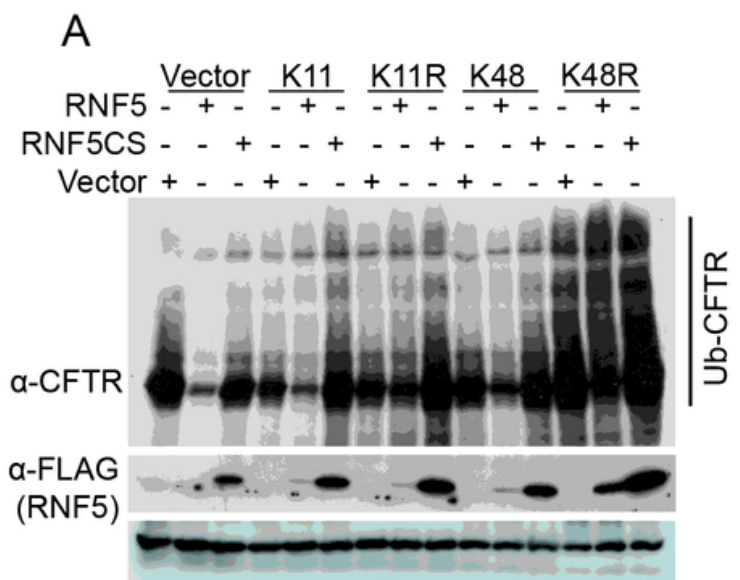

B

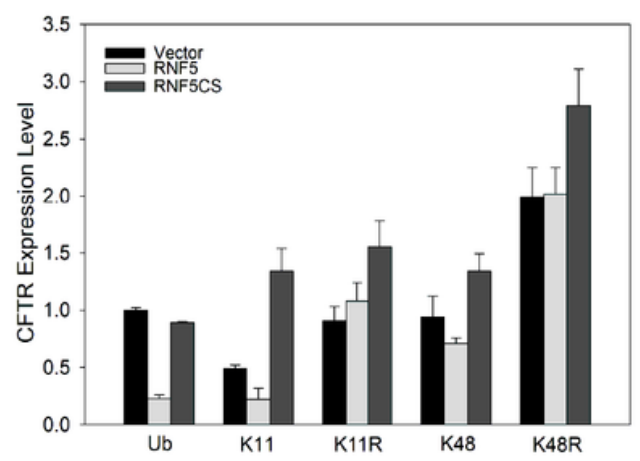

C CHIP $\frac{\text { Vector }}{+-} \frac{\mathrm{K} 11}{-+-} \frac{\mathrm{K} 11 \mathrm{R}}{-+-} \frac{\mathrm{K} 48}{-+} \frac{\mathrm{K} 48 \mathrm{R}}{-+-}$ CHIPdUb - - + - + - + + - + - + Vector $+\ldots+\ldots+\ldots+\ldots+-$

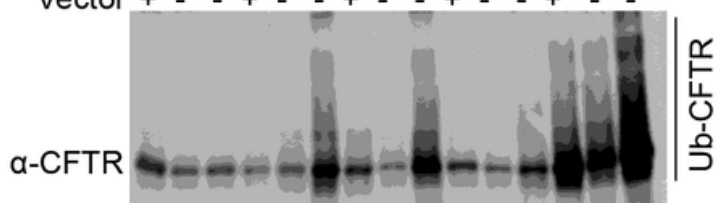

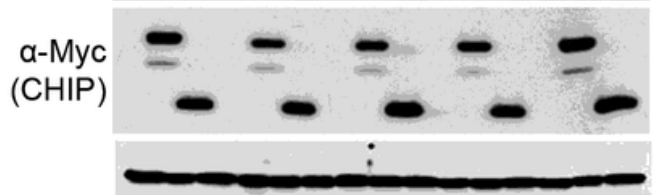

D

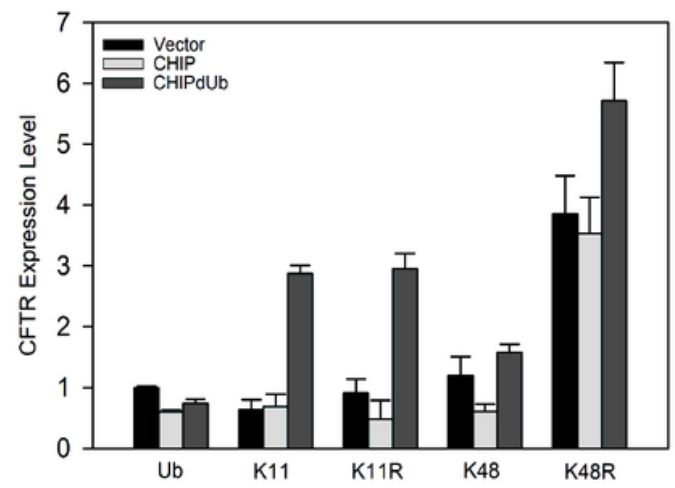

Figure 5

E3 ligases catalyzed PLLCs formation of F508del. A, C, HEK293 cells were transfected with indicated plasmids and collected after 48 hours of transfection and detected using Western blotting. B, D, Quantitation of proteins of panel A or $C($ mean $\pm S E, n=3)$.

A

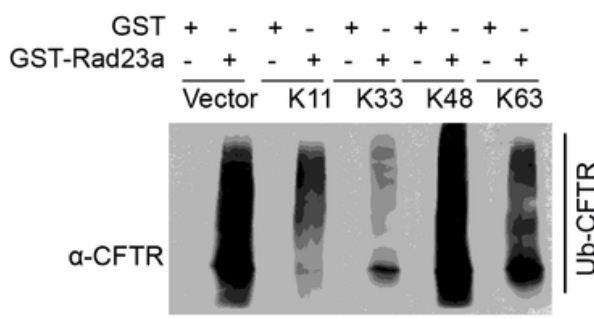

C

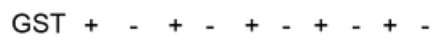

GST-Adrm1 $\frac{-+}{\text { Vector }} \frac{-+}{\mathrm{K} 11} \frac{-+}{\mathrm{K} 33} \frac{-+}{\mathrm{K} 48} \frac{+}{\mathrm{K} 63}$

a-CFTR

\section{$B$}

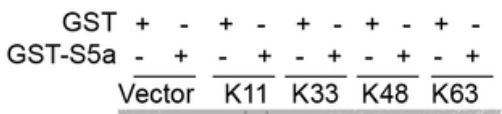

a-CFTR
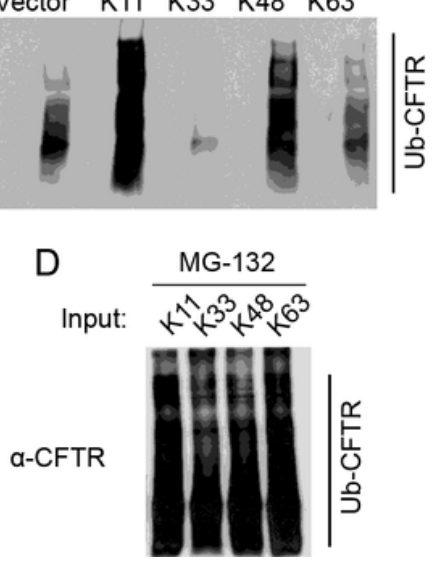

E

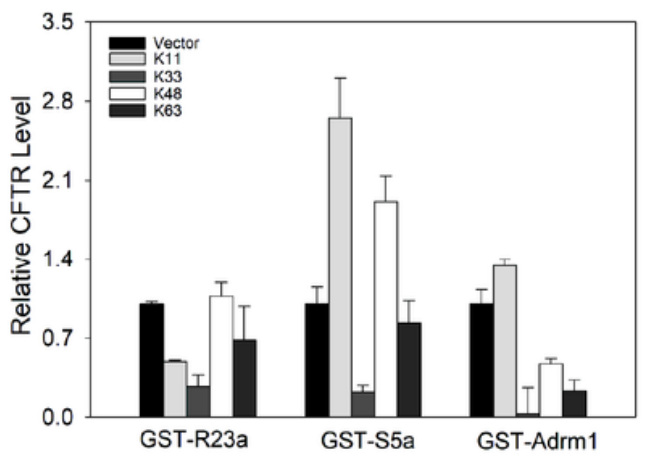

Figure 6 
Physical interaction between ubiquitilyzed F508del and the proteasomal shuttle protein or receptors. A-C, HEK293 cells were transfected with indicated plasmids and collected 48 hours post-transfection and followed by treatment with $10 \mu \mathrm{M}$ MG-132 for 4 hours. CFTR was pull down using purified GST and GSTfusion proteins binding agarose beads from $1 \mathrm{mg}$ total cell lysates. the precipitants were subjected to immunoblotting. D, $50 \mu \mathrm{g}$ total protein from HEK293 cells expressing F508del plus Ub or its mutants with MG-132 treatment.

\section{Supplementary Files}

This is a list of supplementary files associated with this preprint. Click to download.

- Fig.S1.tif

- Fig.S2.tif

- Fig.S3.tif

- Fig.S4.tif 